\title{
Concatenation of Finite Sequences
}

\author{
Rafał Ziobro \\ Department of Carbohydrate Technology \\ University of Agriculture \\ Krakow, Poland
}

\begin{abstract}
Summary. The coexistence of "classical" finite sequences [1] and their zero-based equivalents finite 0-sequences [6] in Mizar has been regarded as a disadvantage. However the suggested replacement of the former type with the latter [5] has not yet been implemented, despite of several advantages of this form, such as the identity of length and domain operators [4]. On the other hand the number of theorems formalized using finite sequence notation is much larger then of those based on finite 0 -sequences, so such translation would require quite an effort.

The paper addresses this problem with another solution, using the Mizar system [3], 2]. Instead of removing one notation it is possible to introduce operators which would concatenate sequences of various types, and in this way allow utilization of the whole range of formalized theorems. While the operation could replace existing FS2XFS, XFS2FS commands (by using empty sequences as initial elements) its universal notation (independent on sequences that are concatenated to the initial object) allows to "forget" about the type of sequences that are concatenated on further positions, and thus simplify the proofs.
\end{abstract}

MSC: 11B99 68T99 03B35

Keywords: finite sequence; finite 0-sequence; concatenation

MML identifier: RVSUM_4, version: 8.1.09 5.54.1341

\section{Preliminaries}

Let $a$ be a real number and $b$ be a non negative real number. One can check that $a-^{\prime}(a+b)$ is zero.

One can check that $a+b-^{\prime} a$ reduces to $b$. 
Let $n, m$ be natural numbers. We identify $n \cap m$ with $\min (m, n)$. We identify $\min (m, n)$ with $n \cap m$. We identify $\max (m, n)$ with $n \cup m$. Let $n, m$ be non negative real numbers. Observe that $\min (n+m, n)$ reduces to $n$ and $\max (n+$ $m, n$ ) reduces to $n+m$.

Now we state the propositions:

(1) Let us consider a binary relation $f$, and natural numbers $n, m$. Then $(f \uparrow(n+m))\lceil n=f\lceil n$.

(2) Let us consider a function $f$, a natural number $n$, and a non zero natural number $m$. Then $(f \uparrow(n+m))(n)=f(n)$.

Let $D$ be a non empty set, $x$ be a sequence of $D$, and $n$ be a natural number. Let us note that $\operatorname{dom}(x\lceil n)$ reduces to $n$. Observe that $x\lceil n$ is finite and transfinite sequence-like and $x\lceil n$ is $n$-element.

\section{Complex-Valued Sequences}

Now we state the proposition:

(3) Let us consider complex-valued functions $f, g$, and a set $X$. Then $(f$. $g)\lceil X=(f\lceil X) \cdot(g\lceil X)$.

Proof: For every object $x$ such that $x \in \operatorname{dom}((f \cdot g) \mid X)$ holds $((f$. $g)\lceil X)(x)=((f\lceil X) \cdot(g\lceil X))(x)$.

Let $D$ be a non empty set and $f, g$ be sequences of $D$. Let us note that $f+\cdot g$ is transfinite sequence-like.

Let $f$ be a constant complex sequence and $n$ be a natural number. Let us note that $f \uparrow n$ is constant and there exists a complex sequence which is empty yielding and there exists a sequence of real numbers which is empty yielding and every complex sequence which is empty yielding is also natural-valued and there exists a complex sequence which is constant and real-valued.

Now we state the proposition:

(4) Let us consider a sequence $s$ of real numbers, and a natural number $n$. Then $\left(\left(\sum_{\alpha=0}^{\kappa} s(\alpha)\right)_{\kappa \in \mathbb{N}}\right)(n)=\sum\left(s \mid \mathbb{Z}_{n+1}\right)$.

Let $c$ be a complex number. The functor $\{c\}_{n \in \mathbb{N}}$ yielding a complex sequence is defined by the term

(Def. 1) $\mathbb{N} \longmapsto c$.

Let $n$ be a natural number. One can check that $\left(\{c\}_{n \in \mathbb{N}}\right)(n)$ reduces to $c$. Now we state the proposition:

(5) Let us consider complex-valued functions $f, g$, and a set $X$. Then $(f+$ $g)\lceil X=f\lceil X+g\lceil X$

Proof: For every object $x$ such that $x \in \operatorname{dom}((f+g)\lceil X)$ holds $((f+$ $g)\lceil X)(x)=(f\lceil X+g \uparrow X)(x)$. 
Let $f$ be a 1-element finite sequence. One can verify that $\langle f(1)\rangle$ reduces to $f$.

Let $f$ be a 2-element finite sequence. Let us note that $\langle f(1), f(2)\rangle$ reduces to $f$.

Let $f$ be a 3 -element finite sequence. Let us note that $\langle f(1), f(2), f(3)\rangle$ reduces to $f$.

Now we state the propositions:

(6) Let us consider a complex-valued finite sequence $f$. Then $\sum f=f(1)+$ $\sum f_{l 1}$.

(7) Let us consider a non empty, complex-valued finite sequence $f$. Then $\prod f=f(1) \cdot\left(\prod f_{l 1}\right)$.

(8) Let us consider a natural number $n$, a non zero natural number $m$, and an $(n+m)$-element finite sequence $f$. Then $f\left\lceil(n+1)=\left(f\lceil n)^{\wedge}\langle f(n+1)\rangle\right.\right.$.

(9) Let us consider a complex-valued finite sequence $f$, and a natural number $n$. Then $\prod f=\prod\left(f\lceil n) \cdot \prod f_{\lfloor n}\right.$.

Proof: Define $\mathcal{P}$ [natural number $] \equiv \prod f=\left(\prod\left(f \backslash \$_{1}\right)\right) \cdot\left(\prod f_{\mid \$_{1}}\right)$. For every natural number $k$ such that $\mathcal{P}[k]$ holds $\mathcal{P}[k+1]$ by $[8,(35)]$, (7). For every natural number $x, \mathcal{P}[x]$.

(10) Let us consider complex-valued finite sequences $f, g$. Then $\prod\left(f^{\frown} g\right)=$ $\left(\prod f\right) \cdot\left(\prod g\right)$. The theorem is a consequence of $(9)$.

\section{On Product and Sum of Complex Sequences}

Let $s$ be a complex sequence. The partial product of $s$ yielding a complex sequence is defined by

(Def. 2) $i t(0)=s(0)$ and for every natural number $n, i t(n+1)=i t(n) \cdot s(n+1)$.

Now we state the propositions:

(11) Let us consider a complex sequence $f$, and a natural number $n$. Suppose $f(n)=0$. Then (the partial product of $f)(n)=0$.

(12) Let us consider a complex sequence $f$, and natural numbers $n, m$. Suppose $f(n)=0$. Then (the partial product of $f)(n+m)=0$.

PROOF: Define $\mathcal{P}$ [natural number] $\equiv($ the partial product of $f)\left(n+\$_{1}\right)=0$. $\mathcal{P}[0]$. For every natural number $k$ such that $\mathcal{P}[k]$ holds $\mathcal{P}[k+1]$. For every natural number $x, \mathcal{P}[x]$.

Let $c$ be a complex number and $n$ be a non zero natural number. Observe that the functor $c^{n}$ is defined by the term

(Def. 3) (the partial product of $\left.\{c\}_{n \in \mathbb{N}}\right)(n-1)$.

Now we state the proposition: 
(13) Let us consider a natural number $n$. Then (the partial product of $\left.\left\{0_{\mathbb{C}}\right\}_{n \in \mathbb{N}}\right)(n)=0$. The theorem is a consequence of (12).

Let $k$ be a natural number. Let us note that (the partial product of $\left.\{0\}_{n \in \mathbb{N}}\right)(k)$ reduces to 0 .

One can verify that every complex sequence which is empty yielding is also absolutely summable and every sequence of real numbers which is empty yielding is also absolutely summable.

Observe that $\left(\sum_{\alpha=0}^{\kappa}(\mathbb{N} \longmapsto 0)(\alpha)\right)_{\kappa \in \mathbb{N}}$ reduces to $\mathbb{N} \longmapsto 0$ and the partial product of $\{0\}_{n \in \mathbb{N}}$ reduces to $\{0\}_{n \in \mathbb{N}}$. One can verify that every complex sequence is transfinite sequence-like and there exists a sequence of $\mathbb{C}$ which is summable.

Let $s_{1}$ be an empty yielding complex sequence. One can check that $\sum s_{1}$ is zero.

Let $s_{1}$ be an empty yielding sequence of real numbers. Let us note that $\sum s_{1}$ is zero.

\section{Finite 0-SEQUences}

Let $c$ be a complex number. Observe that $\langle c\rangle$ is complex-valued.

One can verify that $\sum\langle c\rangle$ reduces to $c$.

Let $n$ be a natural number. One can verify that there exists a natural-valued finite 0 -sequence which is $n$-element.

Let $k$ be an object. One can check that $n \longmapsto k$ is $n$-element and there exists a finite 0 -sequence which is $n$-element.

Let $f$ be an $n$-element finite 0 -sequence. Let us note that $f\lceil n$ reduces to $f$.

Let $n, m$ be natural numbers. One can check that $f \uparrow(n+m)$ reduces to $f$.

Let $f$ be a 1-element finite 0 -sequence. Let us note that $\langle f(0)\rangle$ reduces to $f$.

Let $f$ be a 2-element finite 0 -sequence. Let us note that $\langle f(0), f(1)\rangle$ reduces to $f$.

Let $f$ be a 3 -element finite 0 -sequence. One can verify that $\langle f(0), f(1), f(2)\rangle$ reduces to $f$.

Now we state the propositions:

(14) Let us consider natural numbers $n, k$. If $k \in \mathbb{Z}_{n+1}$, then $n-k$ is a natural number.

(15) Let us consider complex numbers $a, b$, and natural numbers $n, k$. Suppose $k \in \mathbb{Z}_{n+1}$. Then there exists an object $c$ and there exists a natural number $l$ such that $l=n-k$ and $c=a^{l} \cdot\left(b^{k}\right)$. The theorem is a consequence of (14). 


\section{Shifting Sequences}

Let $f$ be a complex-valued finite 0 -sequence and $s_{1}$ be a complex sequence. The functor $f^{\frown} s_{1}$ yielding a complex sequence is defined by the term (Def. 4) $f \cup \operatorname{Shift}\left(s_{1}\right.$, len $\left.f\right)$.

Let $f$ be a function. The functor $s_{1} \frown f$ yielding a sequence of $\mathbb{C}$ is defined by the term

(Def. 5) $s_{1}$.

Now we state the propositions:

(16) Let us consider an object $x$. Then $x$ is a real-valued complex sequence if and only if $x$ is a sequence of real numbers.

(17) Let us consider a sequence $r_{1}$ of real numbers, and a complex sequence $c_{1}$. Suppose $c_{1}=r_{1}$. Then the partial product of $r_{1}=$ the partial product of $c_{1}$.

Let $f$ be a complex-valued finite 0 -sequence and $s_{1}$ be a sequence of real numbers. The functor $f \frown s_{1}$ yielding a complex sequence is defined by the term (Def. 6) $f \cup \operatorname{Shift}\left(s_{1}\right.$, len $\left.f\right)$.

Now we state the proposition:

(18) Let us consider a sequence $r_{1}$ of real numbers. Then \langle\rangle$_{\mathbb{R}} \frown r_{1}$ is a realvalued complex sequence.

Let $f$ be a sequence of real numbers and $g$ be a function. The functor $f \frown g$ yielding a real-valued sequence of $\mathbb{C}$ is defined by the term

(Def. 7) $f$.

Let $f$ be a complex-valued finite 0 -sequence and $s_{1}$ be a complex sequence. Let us observe that $\left(f \frown s_{1}\right)\lceil\operatorname{dom} f$ reduces to $f$.

Let $s_{1}$ be a sequence of real numbers. Let us note that $\left(f \frown s_{1}\right)\lceil\operatorname{dom} f$ reduces to $f$.

Now we state the propositions:

(19) Let us consider a complex-valued finite 0-sequence $f$, and a natural number $x$. Then $\left(f \frown\{0\}_{n \in \mathbb{N}}\right)(x)=f(x)$.

(20) Let us consider a sequence $f$ of real numbers. Then $f \frown f$ is a real-valued complex sequence.

Let $f$ be a real-valued complex sequence. Note that $\Im(f)$ is empty yielding. One can check that $\Re(f)$ reduces to $f$.

Let us observe that there exists a sequence of real numbers which is empty yielding and every sequence of real numbers is transfinite sequence-like.

Let $r$ be a real number. Let us note that $\Re(r \cdot(i))$ is zero.

One can check that $\Im(r \cdot(i))$ reduces to $r$. 
Let $f$ be a complex-valued finite 0 -sequence. Let us note that $\Re(f)$ is realvalued, finite, and transfinite sequence-like and $\Im(f)$ is real-valued, finite, and transfinite sequence-like and $\Re(f)$ is (len $f$ )-element and $\Im(f)$ is (len $f$ )-element.

Let $f$ be a complex-valued finite sequence. Note that $\Re(f)$ is real-valued and finite sequence-like and $\Im(f)$ is real-valued and finite sequence-like.

Let $f$ be a complex-valued function. Let us observe that $\Re(\Re(f))$ reduces to $\Re(f)$ and $\Re(\Im(f))$ reduces to $\Im(f)$. Let us note that $\Im(\Re(f))$ is empty yielding and $\Im(\Im(f))$ is empty yielding.

One can check that $\Re(\Re(f)+i \cdot \Im(f))$ reduces to $\Re(f)$ and $\Im(\Re(f)+i \cdot \Im(f))$ reduces to $\Im(f)$ and $\Re(f)+i \cdot \Im(f)$ reduces to $f$.

Let $n$ be a natural number. One can check that there exists a finite function which is $n$-element.

Let $f$ be a finite, complex-valued transfinite sequence. Note that $\operatorname{Shift}(f, n)$ is finite and $\operatorname{Shift}(f, n)$ is (len $f$ )-element and $\{0\}_{n \in \mathbb{N}}$ is empty yielding.

\section{Converting Complex 0-Sequences into Ordinary Ones}

Let $f$ be a complex-valued finite 0 -sequence. The functor Sequel $f$ yielding a complex sequence is defined by the term

(Def. 8) $(\mathbb{N} \longmapsto 0)+\cdot f$.

Now we state the propositions:

(21) Let us consider a complex-valued finite 0 -sequence $f$, and a natural number $x$. Then (Sequel $f)(x)=f(x)$.

(22) Let us consider a complex-valued finite 0 -sequence $f$. Then Sequel $f=$ $f^{\frown}\{0\}_{n \in \mathbb{N}}$.

Proof: $\operatorname{dom}($ Sequel $f)=\operatorname{dom}\left(f^{\frown}\{0\}_{n \in \mathbb{N}}\right)$. For every natural number $x$, $($ Sequel $f)(x)=\left(f \frown\{0\}_{n \in \mathbb{N}}\right)(x)$.

(23) Let us consider a complex sequence $s_{1}$. Then $s_{1}=\Re\left(s_{1}\right)+i \cdot \Im\left(s_{1}\right)$.

Let us consider a complex-valued finite 0 -sequence $f$. Now we state the propositions:

(24) $\Re($ Sequel $f)=$ Sequel $\Re(f)$. The theorem is a consequence of (21).

(25) $\Im($ Sequel $f)=\operatorname{Sequel} \Im(f)$. The theorem is a consequence of $(21)$.

Now we state the propositions:

(26) Let us consider a complex number $c$. Then $0 \cdot(\mathbb{N} \longmapsto c)=\mathbb{N} \longmapsto 0$.

(27) Let us consider a complex sequence $s_{1}$, and a natural number $x$. Suppose for every natural number $k$ such that $k \geqslant x$ holds $s_{1}(k)=0$. Then $s_{1}$ is summable. 
(28) Let us consider a sequence $s_{1}$ of real numbers, and a natural number $x$. Suppose for every natural number $k$ such that $k \geqslant x$ holds $s_{1}(k)=0$. Then $s_{1}$ is summable.

Let $f$ be a complex-valued finite 0 -sequence. One can check that Sequel $f$ is summable.

\section{Properties of Concatenation}

Let $f$ be a finite 0 -sequence and $g$ be a finite sequence. The functor $f^{\frown} g$ yielding a finite 0 -sequence is defined by

(Def. 9) $\operatorname{dom} i t=\operatorname{len} f+\operatorname{len} g$ and for every natural number $k$ such that $k \in \operatorname{dom} f$ holds it $(k)=f(k)$ and for every natural number $k$ such that $k \in \operatorname{dom} g$ holds it $($ len $f+k-1)=g(k)$.

Let $f$ be a finite sequence and $g$ be a finite 0 -sequence. The functor $f^{\frown} g$ yielding a finite sequence is defined by

(Def. 10) $\operatorname{dom} i t=\operatorname{Seg}(\operatorname{len} f+\operatorname{len} g)$ and for every natural number $k$ such that $k \in \operatorname{dom} f$ holds $i t(k)=f(k)$ and for every natural number $k$ such that $k \in \operatorname{dom} g$ holds it (len $f+k+1)=g(k)$.

Now we state the proposition:

(29) Let us consider a finite 0 -sequence $f$, and a finite sequence $g$. Then

(i) $\operatorname{len}(f \frown g)=\operatorname{len} f+\operatorname{len} g$, and

(ii) $\operatorname{len}(g \frown f)=\operatorname{len} f+\operatorname{len} g$.

Let $n, m$ be natural numbers, $f$ be an $n$-element finite 0 -sequence, and $g$ be an $m$-element finite sequence. Let us note that $f^{\frown} g$ is $(n+m)$-element and $g^{\frown} f$ is $(n+m)$-element.

Now we state the propositions:

(30) Let us consider a finite 0-sequence $f$, a finite sequence $g$, and a natural number $x$. Then $x \in \operatorname{dom}\left(f^{\wedge} g\right)$ if and only if $x \in \operatorname{dom} f$ or $x+1-\operatorname{len} f \in$ $\operatorname{dom} g$.

Proof: If $x \in \operatorname{dom}\left(f^{\wedge} g\right)$, then $x \in \operatorname{dom} f$ or $x+1-\operatorname{len} f \in \operatorname{dom} g$. If $x \in \operatorname{dom} f$ or $x+1-\operatorname{len} f \in \operatorname{dom} g$, then $x \in \operatorname{dom}(f \frown g)$.

(31) Let us consider a finite sequence $f$, a finite 0 -sequence $g$, and a natural number $x$. Then $x \in \operatorname{dom}\left(f^{\wedge} g\right)$ if and only if $x \in \operatorname{dom} f$ or $x-(\operatorname{len} f+1) \in$ $\operatorname{dom} g$.

Proof: If $x \in \operatorname{dom}\left(f^{\frown} g\right)$, then $x \in \operatorname{dom} f$ or $x-(\operatorname{len} f+1) \in \operatorname{dom} g$.

(32) Let us consider a finite sequence $f$, and a finite 0 -sequence $g$. Then

(i) $\operatorname{rng}(f \frown g)=\operatorname{rng} f \cup \operatorname{rng} g$, and 
(ii) $\operatorname{rng}\left(g^{\frown} f\right)=\operatorname{rng} f \cup \operatorname{rng} g$.

Proof: $\operatorname{rng}\left(f^{\frown} g\right) \subseteq \operatorname{rng} f \cup \operatorname{rng} g$. $\operatorname{rng} f \cup \operatorname{rng} g \subseteq \operatorname{rng}\left(f^{\frown} g\right) \cdot \operatorname{rng}\left(g^{\frown} f\right) \subseteq$ $\operatorname{rng} f \cup \operatorname{rng} g$. $\operatorname{rng} f \cup \operatorname{rng} g \subseteq \operatorname{rng}(g \frown f)$.

(33) Let us consider a non empty finite 0 -sequence $f$, and a finite sequence $g$. Then $\operatorname{dom}(f \cup \operatorname{Shift}(g, \operatorname{len} f-1))=\mathbb{Z}_{\text {len } f+\operatorname{len} g}$.

Proof: For every object $x, x \in \operatorname{dom}(f \cup \operatorname{Shift}(g$, len $f-1))$ iff $x \in$ $\mathbb{Z}_{\text {len } f+\text { len } g}$.

(34) Let us consider a finite sequence $f$, and a finite 0 -sequence $g$. Then $\operatorname{dom}(f \cup \operatorname{Shift}(g, \operatorname{len} f+1))=\operatorname{Seg}(\operatorname{len} f+\operatorname{len} g)$.

Proof: For every object $x, x \in \operatorname{dom}(f \cup \operatorname{Shift}(g, \operatorname{len} f+1))$ iff $x \in$ $\operatorname{Seg}(\operatorname{len} f+\operatorname{len} g)$.

Let $f$ be a complex-valued finite sequence. One can verify that \langle\rangle$_{\mathbb{C}}{ }^{\wedge}$ is complex-valued.

Let $f$ be a complex-valued finite 0 -sequence. Let us note that $\left.\varepsilon_{\mathbb{C}}\right\urcorner f$ is complex-valued.

Let $f$ be a finite 0 -sequence and $g$ be a finite sequence. One can verify that $\left(f^{\frown} g\right)\left\lceil\operatorname{len} f\right.$ reduces to $f$ and $\left(g^{\frown} f\right)\lceil$ len $g$ reduces to $g$.

Now we state the propositions:

(35) Let us consider a set $D$, a finite 0 -sequence $f$, and a finite sequence $g$ of elements of $D$. Then $\left(f^{\frown} g\right)_{\text {llen } f}=\operatorname{FS} 2 X F S(g)$.

Proof: For every natural number $i$ such that $i \in \operatorname{dom}\left(\left(f^{\wedge} g\right)_{\mid \text {len } f}\right)$ holds $\left((f \frown g)_{\lfloor\operatorname{len} f}\right)(i)=(\operatorname{FS} 2 X F S(g))(i)$.

(36) Every finite 0 -sequence is a finite 0 -sequence of $\operatorname{rng} f \cup\{1\}$.

(37) Let us consider a set $D$, a finite sequence $f$, and a finite 0 -sequence $g$ of $D$. Then $\left(f^{\frown} g\right)_{\lfloor\text {len } f}=\operatorname{XFS} 2 \mathrm{FS}(g)$.

Proof: len $f \leqslant \operatorname{len}(f \frown g)$. For every natural number $i$ such that $i \in$ $\operatorname{dom}\left((f \frown g)_{\text {llen } f}\right)$ holds $\left((f \frown g)_{\mid \text {len } f}\right)(i)=(\operatorname{XFS} 2 \mathrm{FS}(g))(i)$.

Let $D$ be a set and $f$ be a finite 0 -sequence of $D$. One can verify that the functor $\mathrm{XFS} 2 \mathrm{FS}(f)$ is defined by the term

(Def. 11) $\varepsilon_{D} \frown f$.

Now we state the proposition:

(38) Let us consider a set $D$, and a finite 0 -sequence $f$ of $D$.

Then $\operatorname{dom}(\operatorname{Shift}(f, 1))=\operatorname{Seg}$ len $f$.

Proof: For every object $x$ such that $x \in \operatorname{Seg}$ len $f$ holds $x \in \operatorname{dom}(\operatorname{Shift}(f, 1))$.

For every object $x$ such that $x \in \operatorname{dom}(\operatorname{Shift}(f, 1))$ holds $x \in \operatorname{Seg}$ len $f$ by [7. (106)].

Let $D$ be a set and $f$ be a finite 0 -sequence of $D$. One can verify that the functor $\operatorname{XFS} 2 \mathrm{FS}(f)$ is defined by the term 
(Def. 12) $\operatorname{Shift}(f, 1)$.

Let $f$ be a finite sequence of elements of $D$. One can check that the functor $\operatorname{FS} 2 \mathrm{XFS}(f)$ is defined by the term

(Def. 13) \langle\rangle$_{D} \frown f$.

Now we state the propositions:

(39) Let us consider a set $D$, and finite 0-sequences $f, g$ of $D$. Then $f^{\frown} g=$ $f^{\frown} \operatorname{XFS} 2 \mathrm{FS}(g)$.

PROOF: For every natural number $k$ such that $k \in \operatorname{dom}\left(f^{\frown} g\right)$ holds $(f \frown g)(k)=(f \frown \operatorname{XFS} 2 \mathrm{FS}(g))(k)$.

(40) Let us consider a set $D$, and finite sequences $f, g$ of elements of $D$. Then $f \frown g=f \frown \operatorname{FS} 2 X F S(g)$.

Proof: For every natural number $k$ such that $k \in \operatorname{dom}\left(f^{\frown} g\right)$ holds $(f \frown g)(k)=(f \frown \operatorname{FS} 2 \operatorname{XFS}(g))(k)$.

Let $f$ be a finite 0 -sequence of $\mathbb{R}$. Let us observe that Sequel $f\lceil\operatorname{dom} f$ reduces to $f$. One can check that $\operatorname{Shift}(f, 1)$ is finite sequence-like and Sequel $f \uparrow \operatorname{dom} f$ is empty yielding.

Now we state the propositions:

(41) Let us consider a set $D$, a finite sequence $f$ of elements of $D$, and a finite 0 -sequence $g$ of $D$. Then $f^{\frown} g=f^{\frown} \operatorname{XFS2FS}(g)$. The theorem is a consequence of (40).

(42) Let us consider a set $D$, a finite 0 -sequence $f$ of $D$, and a finite sequence $g$ of elements of $D$. Then $f^{\frown} g=f^{\frown} \operatorname{FS} 2 X F S(g)$. The theorem is a consequence of (39).

(43) Let us consider a set $D$, and finite sequences $f, g$ of elements of $D$. Then $\operatorname{FS} 2 \operatorname{XFS}\left(f^{\frown} g\right)=\operatorname{FS} 2 \operatorname{XFS}(f) \frown \operatorname{FS} 2 \operatorname{XFS}(g)$.

Proof: For every natural number $x$ such that $x \in \operatorname{dom}\left(\operatorname{FS} 2 \operatorname{XFS}\left(f^{\frown} g\right)\right)$ holds $(\operatorname{FS} 2 X F S(f \frown g))(x)=(\operatorname{FS} 2 X F S(f) \frown \operatorname{FS} 2 X F S(g))(x)$.

Let $D$ be a set, $f$ be a finite sequence of elements of $D$, and $g$ be a finite 0 -sequence of $D$. Note that the functor $f^{\frown} g$ yields a finite sequence of elements of $D$. Now we state the propositions:

(44) Let us consider a set $D$, a finite sequence $f$ of elements of $D$, and a finite 0 -sequence $g$ of $D$. Then $\operatorname{FS} 2 \operatorname{XFS}\left(f^{\frown} g\right)=\operatorname{FS} 2 \operatorname{XFS}(f)^{\wedge} g$. The theorem is a consequence of (43) and (40).

(45) Let us consider a set $D$, and finite 0 -sequences $f, g$ of $D$. Then XFS2FS $\left(f^{\wedge}\right.$ $g)=\operatorname{XFS} 2 \mathrm{FS}(f) \frown \operatorname{XFS} 2 \mathrm{FS}(g)$.

Proof: For every natural number $x$ such that $x \in \operatorname{dom}\left(\operatorname{XFS} 2 \mathrm{FS}\left(f^{\frown} g\right)\right)$ holds $(\operatorname{XFS} 2 \mathrm{FS}(f \frown g))(x)=(\operatorname{XFS} 2 \mathrm{FS}(f) \frown \operatorname{XFS} 2 \mathrm{FS}(g))(x)$. 
Let $D$ be a set, $f$ be a finite 0 -sequence of $D$, and $g$ be a finite sequence of elements of $D$. One can check that the functor $f^{\frown} g$ yields a finite 0 -sequence of $D$. Now we state the propositions:

(46) Let us consider a set $D$, a finite 0 -sequence $f$ of $D$, and a finite sequence $g$ of elements of $D$. Then XFS2FS $\left(f^{\wedge} g\right)=\operatorname{XFS} 2 \mathrm{FS}(f)^{\wedge} g$. The theorem is a consequence of (45) and (39).

(47) Let us consider a set $D$, finite 0 -sequences $f, g$ of $D$, and a finite sequence $h$ of elements of $D$. Then

(i) $(f \frown g) \frown h=f \frown(g \frown h)$, and

(ii) $(f \frown h) \frown g=f \frown(h \frown g)$, and

(iii) $(h \frown f) \frown g=h \frown(f \frown g)$.

The theorem is a consequence of (42), (39), (43), (41), and (45).

\section{Sum of Finite 0-Sequences}

Now we state the proposition:

(48) Let us consider a complex-valued finite 0 -sequence $f$. Then $\sum(f\lceil 1)=$ $f(0)$.

Let $n, m$ be natural numbers and $f$ be an $(n+m)$-element finite 0 -sequence. One can verify that $f\lceil n$ is $n$-element. Let $n$ be a natural number and $p$ be an $n$ element, complex-valued finite 0 -sequence. Let us observe that $-p$ is $n$-element and $p^{-1}$ is $n$-element and $p^{2}$ is $n$-element and $|p|$ is $n$-element and $\operatorname{Rev}(p)$ is $n$-element.

Let $m$ be a natural number and $q$ be an $(n+m)$-element, complex-valued finite 0 -sequence. Let us observe that $\operatorname{dom} p \cap \operatorname{dom} q$ reduces to $\operatorname{dom} p$. Note that $p+q$ is $n$-element and $p-q$ is $n$-element and $p \cdot q$ is $n$-element and $p / q$ is $n$-element. Let $p, q$ be $n$-element, complex-valued finite 0 -sequences. Note that $p+q$ is $n$-element and $p-q$ is $n$-element and $p \cdot q$ is $n$-element and $p / q$ is $n$-element. Now we state the propositions:

(49) Let us consider a natural number $n$, and $n$-element, complex-valued finite 0 -sequences $f_{1}, f_{2}$. Then $\sum\left(f_{1}+f_{2}\right)=\sum f_{1}+\sum f_{2}$.

Proof: Define $\mathcal{P}$ [natural number] $\equiv$ for every $\$_{1}$-element, complex-valued finite 0 -sequences $f_{1}, f_{2}, \sum\left(f_{1}+f_{2}\right)=\sum f_{1}+\sum f_{2}$. For every natural number $k$ such that $\mathcal{P}[k]$ holds $\mathcal{P}[k+1]$. For every natural number $k, \mathcal{P}[k]$.

(50) Let us consider a complex number $c$. Then XFS2FS $(\langle c\rangle)=\langle c\rangle$. PROOF: For every natural number $k$ such that $k \in \operatorname{dom}\langle c\rangle$ holds $(\mathrm{XFS} 2 \mathrm{FS}(\langle c\rangle))(k)=\langle c\rangle(k)$. 
(51) Let us consider a finite 0 -sequence $f$ of $\mathbb{R}$. Then $\sum \operatorname{XFS} 2 \mathrm{FS}(f)=\sum f$. The theorem is a consequence of (16).

(52) Let us consider a complex-valued finite 0-sequence $f$. Then $\sum f=$ $\sum \Re(f)+(i) \cdot\left(\sum \Im(f)\right)$. The theorem is a consequence of $(49)$.

(53) Let us consider a complex-valued transfinite sequence $f$, and a natural number $n$. Then

(i) $\Re(\operatorname{Shift}(f, n))=\operatorname{Shift}(\Re(f), n)$, and

(ii) $\Im(\operatorname{Shift}(f, n))=\operatorname{Shift}(\Im(f), n)$.

Let us consider a complex-valued finite 0 -sequence $f$.

(i) XFS2FS $(\Re(f))=\Re(\operatorname{XFS} 2 F S(f))$, and

(ii) $\operatorname{XFS} 2 \mathrm{FS}(\Im(f))=\Im(\operatorname{XFS} 2 \mathrm{FS}(f))$.

(55) $\sum \mathrm{XFS} 2 \mathrm{FS}(f)=\sum f$. The theorem is a consequence of (52), (51), and (53).

(56) Let us consider a finite sequence $f$ of elements of $\mathbb{C}$. Then $\sum \operatorname{FS} 2 X F S(f)=$ $\sum f$. The theorem is a consequence of (55).

(57) Let us consider a real-valued finite 0 -sequence $f$. Then $\sum f=\sum$ Sequel $f$.

Note that there exists a real-valued complex sequence which is summable.

Let $f$ be a summable complex sequence. The functors: $\Re(f)$ and $\Im(f)$ yield summable, real-valued complex sequences. Now we state the propositions:

(58) Let us consider a complex-valued finite 0 -sequence $f$. Then $\sum f=$ $\sum$ Sequel $f$. The theorem is a consequence of (57), (24), (25), and (52).

(59) Let us consider a finite 0 -sequence $f$ of $\mathbb{C}$, and a finite sequence $g$ of elements of $\mathbb{C}$. Then

(i) $\sum(f \frown g)=\sum f+\sum g$, and

(ii) $\sum(g \frown f)=\sum g+\sum f$.

The theorem is a consequence of (39), (56), (40), and (55).

\section{Product of Finite 0-Sequences}

Let $f$ be a finite 0 -sequence. The functor $\prod f$ yielding an element of $\mathbb{C}$ is defined by the term

$($ Def. 14) $\cdot \mathbb{C} \odot f$.

Now we state the proposition:

(60) Let us consider an empty finite 0 -sequence $f$. Then $\prod f=1$.

Let $c$ be a complex number. One can check that $\prod\langle c\rangle$ reduces to $c$. 
(61) Let us consider a natural number $n$, and a complex-valued finite 0sequence $f$. Suppose $n \in \operatorname{dom} f$. Then $\prod\left(f\lceil n) \cdot f(n)=\prod(f\lceil(n+1))\right.$.

(62) Let us consider a natural number $n$, and a complex sequence $f$. Then $\prod\left(f\lceil n) \cdot f(n)=\prod(f \uparrow(n+1))\right.$. The theorem is a consequence of $(61)$.

(63) Let us consider a non empty, complex-valued finite 0 -sequence $f$. Then $\prod(f \nmid 1)=f(0)$.

(64) Let us consider a natural number $n$, and $n$-element, complex-valued finite 0 -sequences $f_{1}, f_{2}$. Then $\prod\left(f_{1} \cdot f_{2}\right)=\left(\prod f_{1}\right) \cdot\left(\prod f_{2}\right)$.

Proof: Define $\mathcal{P}$ [natural number] $\equiv$ for every $\$_{1}$-element, complex-valued finite 0 -sequences $f_{1}, f_{2}, \Pi\left(f_{1} \cdot f_{2}\right)=\left(\prod f_{1}\right) \cdot\left(\prod f_{2}\right)$. For every natural number $k$ such that $\mathcal{P}[k]$ holds $\mathcal{P}[k+1]$. $\mathcal{P}[0]$. For every natural number $k, \mathcal{P}[k]$.

(65) Let us consider a complex sequence $f$, and a natural number $n$. Then $\prod(f \uparrow(n+1))=($ the partial product of $f)(n)$.

Proof: Define $\mathcal{P}$ [natural number $] \equiv \prod\left(f \uparrow\left(\$_{1}+1\right)\right)=$ (the partial product of $f)\left(\$_{1}\right)$. $\mathcal{P}[0]$. For every natural number $k$ such that $\mathcal{P}[k]$ holds $\mathcal{P}[k+1]$. For every natural number $x, \mathcal{P}[x]$.

(66) Let us consider a complex-valued finite 0-sequence $f$.

Then $\prod \mathrm{XFS} 2 \mathrm{FS}(f)=\prod f$.

Proof: Define $\mathcal{P}$ [natural number] $\equiv \prod \operatorname{XFS} 2 \mathrm{FS}\left(f \nmid \$_{1}\right)=\prod\left(f\left\lceil \$_{1}\right) . \mathcal{P}[0]\right.$. For every natural number $k$ such that $\mathcal{P}[k]$ holds $\mathcal{P}[k+1]$. For every natural number $x, \mathcal{P}[x]$.

(67) Let us consider a finite sequence $f$ of elements of $\mathbb{C}$. Then $\prod \operatorname{FS} 2 X F S(f)=$ $\prod f$. The theorem is a consequence of (66).

(68) Let us consider a finite 0 -sequence $f$ of $\mathbb{C}$, and a finite sequence $g$ of elements of $\mathbb{C}$. Then

(i) $\prod(f \frown g)=\left(\prod f\right) \cdot\left(\prod g\right)$, and

(ii) $\prod(g \frown f)=\left(\prod g\right) \cdot\left(\prod f\right)$.

The theorem is a consequence of (66), (46), (10), and (40).

Acknowledgement: Ad Maiorem Dei Gloriam

\section{REFERENCES}

[1] Grzegorz Bancerek and Krzysztof Hryniewiecki. Segments of natural numbers and finite sequences. Formalized Mathematics, 1(1):107-114, 1990.

[2] Grzegorz Bancerek, Czesław Byliński, Adam Grabowski, Artur Korniłowicz, Roman Matuszewski, Adam Naumowicz, and Karol Pakk. The role of the Mizar Mathematical Library for interactive proof development in Mizar. Journal of Automated Reasoning, 61(1):9-32, 2018. dor $10.1007 / \mathrm{s} 10817-017-9440-6$ 
[3] Adam Grabowski, Artur Korniłowicz, and Adam Naumowicz. Four decades of Mizar. Journal of Automated Reasoning, 55(3):191-198, 2015. doi 10.1007/s10817-015-9345-1

[4] Artur Kornilowicz. How to define terms in Mizar effectively. Studies in Logic, Grammar and Rhetoric, 18:67-77, 2009.

[5] Piotr Rudnicki and Andrzej Trybulec. On the integrity of a repository of formalized mathematics. In Andrea Asperti, Bruno Buchberger, and James H. Davenport, editors, Mathematical Knowledge Management, volume 2594 of Lecture Notes in Computer Science, pages 162-174. Springer, Berlin, Heidelberg, 2003. doi 10.1007/3-540-36469-2_13.

[6] Tetsuya Tsunetou, Grzegorz Bancerek, and Yatsuka Nakamura. Zero-based finite sequences Formalized Mathematics, 9(4):825-829, 2001.

[7] Rafał Ziobro. Fermat's Little Theorem via divisibility of Newton's binomial. Formalized Mathematics, 23(3):215-229, 2015. doi 10.1515/forma-2015-0018.

[8] Rafał Ziobro. On subnomials. Formalized Mathematics, 24(4):261-273, 2016. doi: $10.1515 /$ forma-2016-0022.

Accepted February 27, 2019 